\title{
Some symmetric identities involving a sequence of polynomials*
}

\author{
Yuan He \\ Department of Mathematics, \\ Northwest University, Xi'an, Shanxi 710069, P.R. China \\ hyyhe@yahoo.com.cn \\ Wenpeng Zhang \\ Department of Mathematics, \\ Northwest University, Xi'an, Shanxi 710069, P.R. China \\ wpzhang@nwu.edu.cn
}

Submitted: Jun 17, 2009; Accepted: Jan 4, 2010; Published: Jan 14, 2010

Mathematics Subject Classifications: 05A19, 11B68

\begin{abstract}
In this paper we establish some symmetric identities on a sequence of polynomials in an elementary way, and some known identities involving Bernoulli and Euler numbers and polynomials are obtained as particular cases.
\end{abstract}

\section{Introduction}

Let $n \in \mathbb{N}=\{0,1, \ldots\}$, and $\left\{f_{n}(x)\right\}_{n=0}^{\infty}$ be a sequence of polynomials given by

$$
\sum_{n=0}^{\infty} f_{n}(x) \frac{t^{n}}{n !}=F(t) \exp ((x-1 / 2) t), \quad \text { c.f. }[12]
$$

where $F(t)$ is a formal power series. The polynomials $f_{n}(x)$ can be denoted by $B_{n}^{(k)}(x)$, $E_{n}^{(k)}(x), G_{n}^{(k)}(x)$, known as the Bernoulli, Euler and Genocchi polynomials of order $k$, according to whether $F(t)$ in Eq. (1.1) is satisfied as follows (see e.g. [10])

$$
F(t)=\left(\frac{t}{\exp (t)-1}\right)^{k} \exp (t / 2),\left(\frac{2}{\exp (t)+1}\right)^{k} \exp (t / 2),\left(\frac{2 t}{\exp (t)+1}\right)^{k} \exp (t / 2) .
$$

\footnotetext{
${ }^{*}$ This research is supported by Natural Science Foundation (Grant 10671155) of China.
} 
The case $k=1$ in Eq. (1.2) gives the classical Bernoulli, Euler and Genocchi polynomials, respectively. The corresponding Bernoulli, Euler and Genocchi numbers are defined by

$$
B_{n}=B_{n}(0), \quad E_{n}=2^{n} E_{n}(1 / 2), \quad \text { and } \quad G_{n}=G_{n}(0) .
$$

These numbers and polynomials play important roles in many branches of mathematics including combinatorics, number theory, special functions and analysis. Numerous interesting properties and relationships for them can be found in many books and papers on this subject, see for example, $[1,10]$.

In recent years, some authors took interest in some symmetric identities involving Bernoulli and Euler numbers and polynomials. For example, In 2001, inspired by the work of Kaneko [8], Momiyama [9] extended Kaneko's identity by using $p$-adic integral over $\mathbb{Z}_{p}$, and showed that for $m, n \in \mathbb{N}$ and $m+n>0$,

$$
\sum_{k=0}^{m}\left(\begin{array}{c}
m+1 \\
k
\end{array}\right)(n+k+1) B_{n+k}+(-1)^{m+n} \sum_{k=0}^{n}\left(\begin{array}{c}
n+1 \\
k
\end{array}\right)(m+k+1) B_{m+k}=0 .
$$

In 2003, by using umbral calculus, Gessel [6] gave another generalization of Kaneko's identity, and obtained that for $m, n \in \mathbb{N}$,

$$
\sum_{k=0}^{m}\left(\begin{array}{l}
m \\
k
\end{array}\right) B_{n+k}+(-1)^{m+n-1} \sum_{k=0}^{n}\left(\begin{array}{l}
n \\
k
\end{array}\right) B_{m+k}=0 .
$$

A generalization for Eq. (1.4) and Eq. (1.5) can be found in [2] (also see [10]). In 2004, $\mathrm{Wu}$, Sun and Pan [12] considered Eq. (1.1) and derived that for $m, n \in \mathbb{N}$,

$$
\sum_{k=0}^{m}\left(\begin{array}{l}
m \\
k
\end{array}\right) f_{n+k}(x)+(-1)^{m+n-1} \sum_{k=0}^{n}\left(\begin{array}{l}
n \\
k
\end{array}\right) f_{m+k}(-x)=0
$$

by which they extended the results of Momiyama and Gessel. After that, Sun [11] researched a sequence of complex numbers and further extended the results in [12]. Meanwhile, he also deduced that for $m, n \in \mathbb{N}$ and $x+y+z=1$,

$$
\sum_{k=0}^{m}\left(\begin{array}{c}
m \\
k
\end{array}\right) x^{m-k} \frac{g_{n+k+1}(y)}{n+k+1}+(-1)^{m+n} \sum_{k=0}^{n}\left(\begin{array}{l}
n \\
k
\end{array}\right) x^{n-k} \frac{g_{m+k+1}(z)}{m+k+1}=\frac{(-1)^{n+1} x^{m+n+1}}{(m+n+1)\left(\begin{array}{c}
m+n \\
m
\end{array}\right)}
$$

where $g_{n}(x)$ denotes Bernoulli polynomials $B_{n}(x)$ or Euler polynomials $E_{n}(x)$; the case where $g_{n}(x)$ denotes Bernoulli polynomials $B_{n}(x)$ and $x=1, y=z=0$ is due to Gelfand [5]. For some applications of Sun's results, we refer to [4, 7]. In 2009, Chen and Sun [3] presented a computer algebra approach to prove the above authors' results by using the extended Zeilberger's algorithm, and they also gave a new result

$$
\sum_{k=0}^{m+3}\left(\begin{array}{c}
m+3 \\
k
\end{array}\right)(m+k+3)(m+k+2)(m+k+1) B_{m+k}=0 .
$$


Inspired by the above work, in this paper we generalize the above authors' results in an elementary way, and obtain that

Theorem 1.1 Let $m, n \in \mathbb{N}$. Then we have

$$
\sum_{k=0}^{m}\left(\begin{array}{l}
m \\
k
\end{array}\right) x^{m-k} f_{n+k}(y)=\sum_{k=0}^{n}\left(\begin{array}{l}
n \\
k
\end{array}\right)(-x)^{n-k} f_{m+k}(x+y) .
$$

Corollary 1.1 Let $m, n \in \mathbb{N}$. Then for any non-negative integer $r$,

$$
\begin{aligned}
& \sum_{k=0}^{m+r}\left(\begin{array}{c}
m+r \\
k
\end{array}\right)\left(\begin{array}{c}
n+r+k \\
r
\end{array}\right) x^{m+r-k} f_{n+k}(y) \\
& =\sum_{k=0}^{n+r}\left(\begin{array}{c}
n+r \\
k
\end{array}\right)\left(\begin{array}{c}
m+r+k \\
r
\end{array}\right)(-x)^{n+r-k} f_{m+k}(x+y) .
\end{aligned}
$$

Proof. By comparing the coefficients of $t^{n+1} /(n+1)$ ! in Eq. (1.1), one can easily obtain $\frac{\mathrm{d}}{\mathrm{d} x} f_{n+1}(x)=(n+1) f_{n}(x)$. Substituting $n+r$ for $n$ and $m+r$ for $m$ in Theorem 1.1, and then making $r$-th derivative for $f_{n+r+k}(y)$ and $f_{m+r+k}(x+y)$ with respect to $y$, respectively, the desired result follows immediately.

Corollary 1.2 Let $m, n \in \mathbb{N}$. Then for any non-negative integer $r$ and $x+y+z=1$,

$$
\begin{aligned}
& \sum_{k=0}^{m+r}\left(\begin{array}{c}
m+r \\
k
\end{array}\right)\left(\begin{array}{c}
n+r+k \\
r
\end{array}\right) x^{m+r-k} g_{n+k}(y) \\
& +(-1)^{m+n+r-1} \sum_{k=0}^{n+r}\left(\begin{array}{c}
n+r \\
k
\end{array}\right)\left(\begin{array}{c}
m+r+k \\
r
\end{array}\right) x^{n+r-k} g_{m+k}(z)=0,
\end{aligned}
$$

where $g_{n}(x)$ denotes Bernoulli polynomials $B_{n}(x)$ or Euler polynomials $E_{n}(x)$. Proof. By comparing the coefficients of $t^{n} / n$ ! in Eq. (1.1), it is easy to see that

$$
f_{n}(1-x)= \begin{cases}(-1)^{n} f_{n}(x), & \text { if } F(t) \text { is an even function, } \\ (-1)^{n+1} f_{n}(x), & \text { if } F(t) \text { is an odd function. }\end{cases}
$$

This together with Corollary 1.1 yields the desired result.

Corollary 1.3 Let $m \in \mathbb{N}$. Then for odd integer $r$,

$$
\sum_{k=0}^{m+r}\left(\begin{array}{c}
m+r \\
k
\end{array}\right)\left(\begin{array}{c}
m+r+k \\
r
\end{array}\right) B_{m+k}=0, \quad \sum_{k=0}^{m+r}\left(\begin{array}{c}
m+r \\
k
\end{array}\right)\left(\begin{array}{c}
m+r+k \\
r
\end{array}\right) E_{m+k}(0)=0 .
$$

Proof. Putting $m=n, x=1$ and $y=z=0$ in Corollary 1.2, the results follow.

Theorem 1.2 Let $m, n \in \mathbb{N}$. Then for any positive integer $r$,

$$
\begin{aligned}
& \sum_{k=0}^{m}\left(\begin{array}{l}
m \\
k
\end{array}\right) x^{m-k} \frac{f_{n+k+r}(y)}{(n+k+1)_{r}} \\
& =\sum_{k=0}^{n}\left(\begin{array}{l}
n \\
k
\end{array}\right)(-x)^{n-k} \frac{f_{m+k+r}(x+y)}{(m+k+1)_{r}}+\frac{(-1)^{n+1} x^{m+n+1}}{(r-1) !} \int_{0}^{1} t^{m}(1-t)^{n} f_{r-1}(x+y-x t) d t,
\end{aligned}
$$


where $(m)_{r}=m(m+1) \cdots(m+r-1)$.

Corollary $1.4[11$, Theorem 1.2] Let $m, n \in \mathbb{N}$. Then for $x+y+z=1$,

$$
\sum_{k=0}^{m}\left(\begin{array}{c}
m \\
k
\end{array}\right) x^{m-k} \frac{g_{n+k+1}(y)}{n+k+1}+(-1)^{m+n} \sum_{k=0}^{n}\left(\begin{array}{l}
n \\
k
\end{array}\right) x^{n-k} \frac{g_{m+k+1}(z)}{m+k+1}=\frac{(-1)^{n+1} x^{m+n+1}}{(m+n+1)\left(\begin{array}{c}
m+n \\
m
\end{array}\right)} .
$$

where $g_{n}(x)$ denotes Bernoulli polynomials $B_{n}(x)$ or Euler polynomials $E_{n}(x)$. Proof. Since $B_{0}(x)=E_{0}(x)=1$ and

$$
\begin{aligned}
\int_{0}^{1} t^{m}(1-t)^{n} d t & =B(m+1, n+1)=\frac{\Gamma(m+1) \cdot \Gamma(n+1)}{\Gamma(m+n+2)} \\
& =\frac{m ! n !}{(m+n+1) !}=\frac{1}{(m+n+1)\left(\begin{array}{c}
m+n \\
m
\end{array}\right)},
\end{aligned}
$$

where $B, \Gamma$ denotes Beta function and Gamma function, respectively, then taking $r=1$ in Theorem 1.2 the result follows from Eq. (1.9).

\section{The proof of Theorem 1.1}

Before proving Theorem 1.1, we need a useful (and obvious) lemma:

Lemma 2.1 Let $\{f(n)\},\{g(n)\},\{h(n)\},\{\bar{h}(n)\}$ be sequences, and

$$
F(t)=\sum_{n=0}^{\infty} f(n) \frac{t^{n}}{n !}, \quad G(t)=\sum_{n=0}^{\infty} g(n) \frac{t^{n}}{n !}, \quad H(t)=\sum_{n=0}^{\infty} h(n) \frac{t^{n}}{n !}, \quad \bar{H}(t)=\sum_{n=0}^{\infty} \bar{h}(n) \frac{t^{n}}{n !},
$$

where $H(t) \bar{H}(t)=1$, then we have

$$
f(n)=\sum_{k=0}^{n}\left(\begin{array}{l}
n \\
k
\end{array}\right) h(k) g(n-k) \Longleftrightarrow g(n)=\sum_{k=0}^{n}\left(\begin{array}{l}
n \\
k
\end{array}\right) \bar{h}(k) f(n-k) .
$$

Next, we give the proof of Theorem 1.1:

Proof. Clearly,

$$
\sum_{m=0}^{\infty}\left[\sum_{k=0}^{m}\left(\begin{array}{l}
m \\
k
\end{array}\right) x^{m-k} f_{n+k}(y)\right] \frac{t^{m}}{m !}=\left[\sum_{m=0}^{\infty} f_{n+m}(y) \frac{t^{m}}{m !}\right] \exp (x t) .
$$

Now, let $f(y, t)=\sum_{m=0}^{\infty} f_{m}(y) \frac{t^{m}}{m !}$, then $\frac{\mathrm{d}^{n}}{\mathrm{~d} t^{n}} f(y, t)=\sum_{m=0}^{\infty} f_{n+m}(y) \frac{t^{m}}{m !}$. It follows from Eq. (2.2) that

$$
\sum_{m=0}^{\infty}\left[\sum_{k=0}^{m}\left(\begin{array}{c}
m \\
k
\end{array}\right) x^{m-k} f_{n+k}(y)\right] \frac{t^{m}}{m !}=\left(\frac{\mathrm{d}^{n}}{\mathrm{~d} t^{n}} f(y, t)\right) \exp (x t) .
$$

On the other hand, since the fact $\exp (-x t) \exp (x t)=1$, and for any $n$-times differentiable function $g(y, t)$,

$$
\left(\frac{\mathrm{d}^{n}}{\mathrm{~d} t^{n}} g(y, t) \exp (x t)\right)=\sum_{k=0}^{n}\left(\begin{array}{l}
n \\
k
\end{array}\right) x^{n-k}\left(\frac{\mathrm{d}^{k}}{\mathrm{~d} t^{k}} g(y, t)\right) \exp (x t) \quad \text { (with Leibniz rule) }
$$


then by Lemma 2.1 we obtain

$$
\left(\frac{\mathrm{d}^{n}}{\mathrm{~d} t^{n}} g(y, t)\right) \exp (x t)=\sum_{k=0}^{n}\left(\begin{array}{l}
n \\
k
\end{array}\right)(-x)^{n-k}\left(\frac{\mathrm{d}^{k}}{\mathrm{~d} t^{k}} g(y, t) \exp (x t)\right) .
$$

So from Eq. (1.1) and Eq. (2.5) we get

$$
\left(\frac{\mathrm{d}^{n}}{\mathrm{~d} t^{n}} f(y, t)\right) \exp (x t)=\sum_{m=0}^{\infty}\left[\sum_{k=0}^{n}\left(\begin{array}{l}
n \\
k
\end{array}\right)(-x)^{n-k} f_{m+k}(x+y)\right] \frac{t^{m}}{m !} .
$$

Equating Eq. (2.3) and Eq. (2.6), we complete the proof of Theorem 1.1 by comparing the coefficients of $t^{m} / m$ !.

\section{The proof of Theorem 1.2}

Lemma 3.1 Let $n \in \mathbb{N}$, then we have

$$
f_{n}(x+y)=\sum_{k=0}^{n}\left(\begin{array}{l}
n \\
k
\end{array}\right) f_{k}(y) x^{n-k}
$$

Proof. Applying the series expansion $\exp (x t)=\sum_{n=0}^{\infty} x^{n} t^{n} / n !$ in Eq. (1.1), the desired result follows immediately.

Next, we give the proof of Theorem 1.2:

Proof. Clearly,

$$
\sum_{m=0}^{\infty}\left[\sum_{k=0}^{m}\left(\begin{array}{c}
m \\
k
\end{array}\right) x^{m-k} \frac{f_{n+k+r}(y)}{(n+k+1)_{r}}\right] \frac{t^{m}}{m !}=\left[\sum_{m=0}^{\infty} \frac{f_{n+m+r}(y)}{(n+m+1)_{r}} \frac{t^{m}}{m !}\right] \exp (x t) .
$$

Let $f(y, t)=\sum_{m=0}^{\infty} \frac{f_{m+r}(y)}{(m+1)_{r}} \frac{t^{m}}{m !}$, then $\frac{\mathrm{d}^{n}}{\mathrm{~d} t^{n}} f(y, t)=\sum_{m=0}^{\infty} \frac{f_{n+m+r}(y)}{(n+m+1)_{r}} \frac{t^{m}}{m !}$. It follows from Eq. (3.1) that

$$
\sum_{m=0}^{\infty}\left[\sum_{k=0}^{m}\left(\begin{array}{l}
m \\
k
\end{array}\right) x^{m-k} \frac{f_{n+k+r}(y)}{(n+k+1)_{r}}\right] \frac{t^{m}}{m !}=\left(\frac{\mathrm{d}^{n}}{\mathrm{~d} t^{n}} f(y, t)\right) \exp (x t) .
$$

Substituting $k$ for $m+r$ in $f(y, t)$, we have

$$
f(y, t)=\sum_{k=r}^{\infty} \frac{f_{k}(y)}{(k-r+1)_{r}} \frac{t^{k-r}}{(k-r) !}=\sum_{m=r}^{\infty} f_{m}(y) \frac{t^{m-r}}{m !} .
$$

So from Eq. (1.1) we obtain

$$
f(y, t)=\frac{F(t) \exp ((y-1 / 2) t)}{t^{r}}-\sum_{i=0}^{r-1} f_{i}(y) \frac{t^{i-r}}{i !} .
$$


Multiplying $\exp (x t)$ in both sides of Eq. (3.4), it follows from Eq. (1.1) that

$$
f(y, t) \exp (x t)=\sum_{m=0}^{\infty} f_{m}(x+y) \frac{t^{m-r}}{m !}-\sum_{i=0}^{r-1} \frac{f_{i}(y)}{i !} \sum_{m=0}^{\infty} x^{m} \frac{t^{m-(r-i)}}{m !} .
$$

The key idea now is to split the right-hand side of Eq. (3.5) into $M_{1}+M_{2}-M_{3}-M_{4}$, where

$$
\begin{gathered}
\mathrm{M}_{1}=\sum_{m=0}^{r-1} f_{m}(x+y) \frac{t^{m-r}}{m !}, \quad \mathrm{M}_{2}=\sum_{m=r}^{\infty} f_{m}(x+y) \frac{t^{m-r}}{m !} \\
\mathrm{M}_{3}=\sum_{i=0}^{r-1} \frac{f_{i}(y)}{i !} \sum_{m=0}^{r-i-1} x^{m} \frac{t^{m-(r-i)}}{m !}, \quad \mathrm{M}_{4}=\sum_{i=0}^{r-1} \frac{f_{i}(y)}{i !} \sum_{m=r-i}^{\infty} x^{m} \frac{t^{m-(r-i)}}{m !} .
\end{gathered}
$$

Note that in a similar consideration to Eq. (3.3) we have

$$
\mathrm{M}_{2}=\sum_{m=0}^{\infty} \frac{f_{m+r}(x+y)}{(m+1)_{r}} \frac{t^{m}}{m !}, \quad \mathrm{M}_{4}=\sum_{i=0}^{r-1} \frac{f_{i}(y)}{i !} \sum_{m=0}^{\infty} \frac{x^{m+r-i}}{(m+1)_{r-i}} \frac{t^{m}}{m !},
$$

and $\mathrm{M}_{3}$ can be reduced in the following way

$$
\mathrm{M}_{3}=\sum_{i=0}^{r-1} \frac{f_{i}(y)}{i !} \sum_{m=i}^{r-1} x^{m-i} \frac{t^{m-r}}{(m-i) !}=\sum_{m=0}^{r-1}\left[\sum_{i=0}^{m}\left(\begin{array}{c}
m \\
i
\end{array}\right) f_{i}(y) x^{m-i}\right] \frac{t^{m-r}}{m !} .
$$

Combining Eq. (3.5)-Eq. (3.7) it follows from Lemma 3.1 that

$$
f(y, t) \exp (x t)=\sum_{m=0}^{\infty}\left[\frac{f_{m+r}(x+y)}{(m+1)_{r}}-\sum_{i=0}^{r-1} \frac{f_{i}(y) x^{m+r-i}}{i !(m+1)_{r-i}}\right] \frac{t^{m}}{m !} .
$$

Thus, by Eq. (2.5) and Eq. (3.8) we derive

$$
\begin{aligned}
\left(\frac{\mathrm{d}^{n}}{\mathrm{~d} t^{n}} f(y, t)\right) \exp (x t)= & \sum_{m=0}^{\infty}\left[\sum_{k=0}^{n}\left(\begin{array}{l}
n \\
k
\end{array}\right)(-x)^{n-k} \frac{f_{m+k+r}(x+y)}{(m+k+1)_{r}}+(-1)^{n+1} x^{m+n+1} \times\right. \\
& \left.\sum_{i=0}^{r-1} x^{r-1-i}\left(\sum_{k=0}^{n}\left(\begin{array}{l}
n \\
k
\end{array}\right)(-1)^{k} \frac{1}{(m+k+1)_{r-i}}\right) \frac{f_{i}(y)}{i !}\right] \frac{t^{m}}{m !},
\end{aligned}
$$

In view of the properties of Beta and Gamma functions used in Eq. (1.10), we have

$$
\sum_{k=0}^{n}\left(\begin{array}{l}
n \\
k
\end{array}\right)(-1)^{k} \frac{1}{(m+k+1)_{r-i}}=\frac{1}{(r-1-i) !} \int_{0}^{1} t^{m}(1-t)^{n}(1-t)^{r-1-i} d t .
$$

It follows from Lemma 3.1 that

$$
\begin{aligned}
& \sum_{i=0}^{r-1} x^{r-1-i}\left(\sum_{k=0}^{n}\left(\begin{array}{l}
n \\
k
\end{array}\right)(-1)^{k} \frac{1}{(m+k+1)_{r-i}}\right) \frac{f_{i}(y)}{i !} \\
& =\frac{1}{(r-1) !} \int_{0}^{1} t^{m}(1-t)^{n}\left[\sum_{i=0}^{r-1}\left(\begin{array}{c}
r-1 \\
i
\end{array}\right) f_{i}(y)(x-x t)^{r-1-i}\right] d t \\
& =\frac{1}{(r-1) !} \int_{0}^{1} t^{m}(1-t)^{n} f_{r-1}(x+y-x t) d t .
\end{aligned}
$$


So from Eq. (3.9) and Eq. (3.10), we have

$$
\begin{aligned}
\left(\frac{\mathrm{d}^{n}}{\mathrm{~d} t^{n}} f(y, t)\right) \exp (x t)= & \sum_{m=0}^{\infty}\left[\sum_{k=0}^{n}\left(\begin{array}{l}
n \\
k
\end{array}\right)(-x)^{n-k} \frac{f_{m+k+r}(x+y)}{(m+k+1)_{r}}+\frac{(-1)^{n+1} x^{m+n+1}}{(r-1) !} \times\right. \\
& \left.\int_{0}^{1} t^{m}(1-t)^{n} f_{r-1}(x+y-x t) d t\right] \frac{t^{m}}{m !}
\end{aligned}
$$

Equating Eq. (3.2) and Eq. (3.11), and comparing the coefficients of $t^{m} / m$ ! we complete the proof of Theorem 1.2.

Acknowledgement. The authors express their gratitude to the referee for his or her helpful comments and suggestions in improving this paper.

\section{References}

[1] M. Abramowitz, I.A. Stegun, Handbook of Mathematical Functions with Formulas Graphs and Mathematical Tables, National Bureau of Standards, Washington, DC, 1964.

[2] T. Agoh, Recurrences for Bernoulli and Euler polynomials and numbers, Exp. Math. 18 (2000), 197-214.

[3] W.Y.C. Chen, L.H. Sun, Extended Zeilberger's algorithm for identities on Bernoulli and Euler polynomials, J. Number Theory 129 (2009), 2111-2132.

[4] W.C. Chu, P. Magli, Summation formulae on reciprocal sequences, European J. Combin. 28 (2007), 921-930.

[5] M.B. Gelfand, A note on a certain relation among Bernoulli numbers, (in Russian), Baškir. Gos. Univ. Učen. Zap. Vyp. 31 (1968), 215-216.

[6] I.M. Gessel, Applications of the classical umbral calculus, Algebra Universalis 49 (2003), 397-434.

[7] J.X. Hou, J. Zeng, A q-analog of dual sequences with applications, European J. Combin. 28 (2007), 214-227.

[8] M. Kaneko, A recurrence formula for the Bernoulli numbers, Proc. Japan Acad. Ser. A Math. Sci. 71 (1995), 192-193.

[9] H. Momiyama, A new recurrence formula for Bernoulli numbers, Fibonacci Quart. 39 (2001), 285-288.

[10] J. Sándor, B. Crstici, Handbook of Number Theory II, Kluwer Academic Publishers, 2004.

[11] Z.W. Sun, Combinatorial identities in dual sequences, European J. Combin. 24 (2003), 709-718.

[12] K.J. Wu, Z.W. Sun, H. Pan, Some identities for Bernoulli and Euler polynomials, Fibonacci Quart. 42 (2004), 295-299. 